\title{
Papers
}

\section{Genetic relation of lobular carcinoma in situ, ductal carcinoma in situ, and associated invasive carcinoma of the breast}

H Buerger, R Simon, K-L Schäfer, R Diallo, R Littmann, C Poremba, P J van Diest, B Dockhorn-Dworniczak, W Böcker

\begin{abstract}
Aims-The mutual relation of lobular carcinoma in situ (LCIS) and ductal carcinoma in situ (DCIS) of the breast, as accepted precursor lesions of invasive breast cancer, is controversial. Because they display genetic heterogeneity, it is not clear how genetically advanced these entities are and what causes the transition to an invasive carcinoma.

Methods-Six cases of LCIS, four of them with associated lobular invasive carcinoma, four cases of intermediately differentiated DCIS with an associated invasive lobular carcinoma, and nine cases of intermediately and poorly differentiated DCIS with associated ductal invasive carcinoma were investigated by means of comparative genomic hybridisation (CGH) after microdissection and immunohistochemical staining of E-cadherin.
\end{abstract}

Results-LCIS was characterised by a low average rate of copy number changes, no evidence of amplifications, and a high rate of gains and losses of chromosomal material at $1 \mathrm{q}$ and $16 \mathrm{q}$, respectively. A high degree of genetic homology with well differentiated DCIS was obvious, as reported previously. The cases of intermediately differentiated DCIS with associated lobular invasive components and lobular differentiation revealed striking homologies, and a significant difference of E-cadherin expression. The comparison of preinvasive and invasive breast lesions, irrespective of differentiation within the same patient, revealed no specific alteration that might be associated with invasion. Genetic alterations seen in invasive carcinoma were not necessarily seen in the adjacent precursor lesions.

Conclusions-These results provide strong evidence that invasive breast cancer is a disease with multiple cytogenetic subclones already present in preinvasive lesions. Moreover, specific CGH alterations associated with invasion were not observed. Furthermore, the close genetic association between well differentiated and a subgroup of intermediately differentiated DCIS and
LCIS led to the hypothesis that LCIS and a subgroup of DCIS are different phenotypic forms of a common genotype.

(F Clin Pathol: Mol Pathol 2000;53:118-121)

Keywords: lobular carcinoma in situ; ductal carcinoma in situ; invasion

The fact that genetic heterogeneity is a hallmark of invasive breast cancer is reflected by the wide spectrum of histological types and differentiation grades in this tumour entity. ${ }^{1}$ Ductal carcinoma in situ (DCIS) of the breast and lobular carcinoma in situ (LCIS) have been proposed to be direct precursors of several subgroups of invasive breast cancer. In a recently published study using comparative genomic hybridisation (CGH), we were able to show that DCIS is a genetically advanced entity and, furthermore, that there are at least two genetic pathways along which DCIS can evolve. ${ }^{2}$ In addition, it was shown that most DCIS and LCIS cases share a common clonal origin with the associated invasive breast cancer. ${ }^{34}$ However, the exact genetic changes associated with invasion remained unclear.

Morphologically, DCIS resembles, and often coincides with, ductal and related (tubular, cribriform, mucinous) types of invasive breast cancer, and LCIS accompanies lobular invasive carcinomas, suggesting close relations with regard to progression. Nevertheless, it is of interest that $15-25 \%$ of cases of DCIS are associated with invasive lobular carcinoma and that an equal proportion of patients diagnosed with LCIS will develop an invasive carcinoma of the ductal subtype over the next 15-20 years. ${ }^{5}$ The putative association between those subtypes is nevertheless a matter of debate. A major difference between ductal and lobular cancer is the expression pattern of E-cadherin, which is almost completely absent in lobular invasive carcinoma and LCIS. ${ }^{6}$

Using a light microscope based microdissection technique and subsequent $\mathrm{CGH}$ analysis, we investigated 39 samples from 17 patients with invasive and preinvasive ductal and lobular breast lesions to establish the role of genetic alterations associated with invasion. The association between LCIS, DCIS, and associated
14 March 2000

email:

boeckew@uni-muenster.de 
invasive carcinoma is discussed in the context of a recently proposed progression model.

\section{Material and methods}

Standard protocols were used in classifying breast neoplasms. ${ }^{78}$ A total of four LCIS cases with associated lobular invasive carcinoma, two isolated LCIS cases, four intermediately differentiated DCIS cases with an associated inva- sive lobular carcinoma, and seven cases of intermediately and poorly differentiated DCIS with associated ductal invasive carcinoma grades 2 or 3 were investigated.

Microdissection was performed under a light microsope, using at least $2510 \mu \mathrm{m}$ thick sections after haematoxylin staining. Wherever possible, all tumour components were microdissected from the same tumour block.
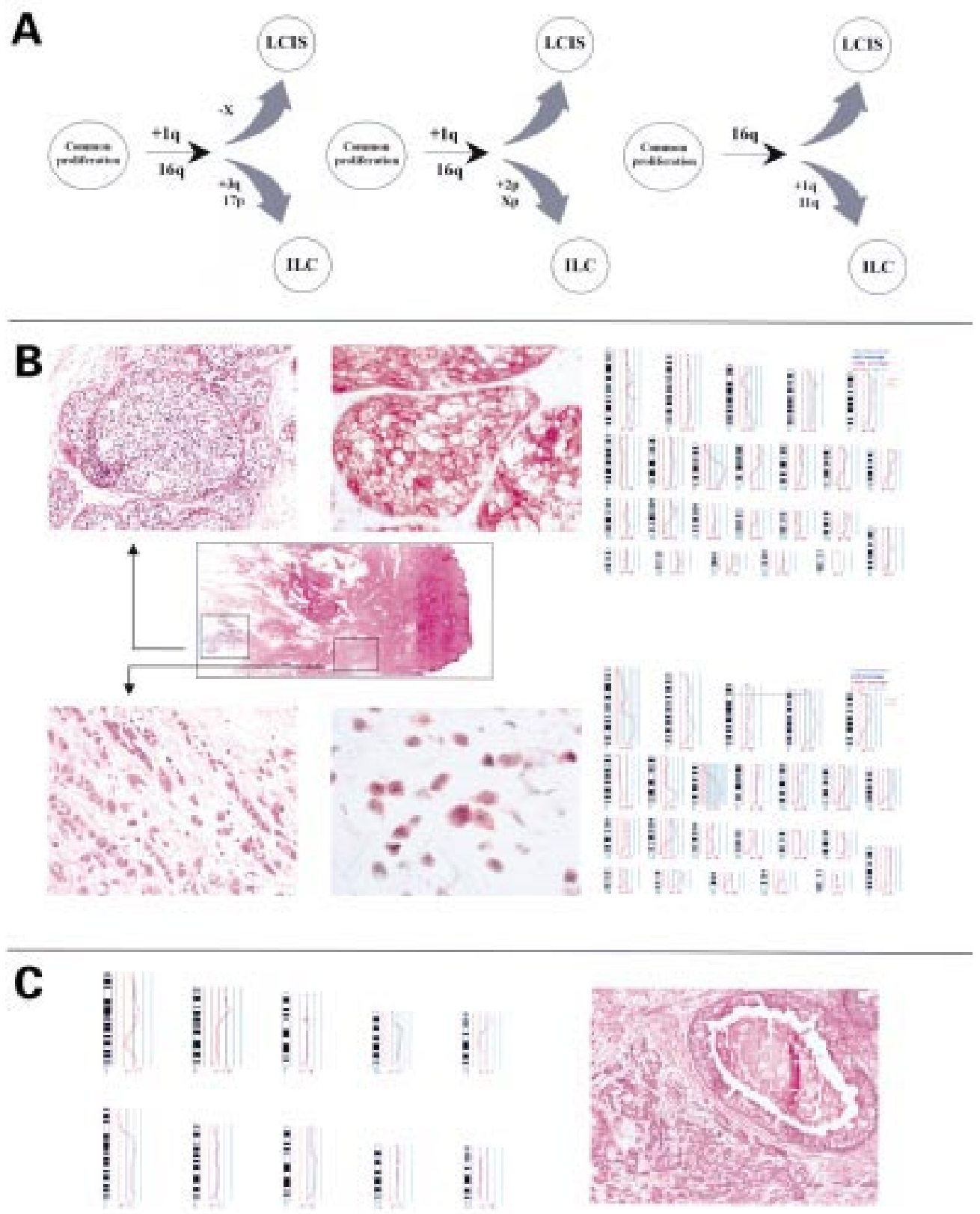

Figure 1 (A) Three examples of hypothetical models of progression of lobular carcinoma in situ (LCIS) and lobular invasive carcinomas. (B) Comparative genomic hybridisation (CGH) ratio profiles, morphology, and immunohistochemistry of intermediately differentiated ductal carcinoma in situ (DCIS) and lobular invasive carcinomas. Middle row left, overview of the section microdissected. The areas of interest are indicated. Upper row left, intermediately differentiated DCIS; haematoxylin and eosin stained; magnification, $\times 10$. Middle, E-cadherin immunohistochemistry; magnification, $\times 10$. Upper row right, CGH ratio profile of intermediately differentiated DCIS. Lower row left, lobular invasive carcinoma; haematoxylin and eosin stained; magnification, $\times 40$. Lower row central, E-cadherin immunohistochemistry; magnification, $\times 40$. Lower row right, $C G H$ ratio profile of lobular invasive carcinoma. The black central line indicates the fluorescence ratio of balanced DNA sequence copy number state (1.0) between tumour and reference DNA. The red lines to the left represent the 0.75 and 0.5 thresholds for losses, the green lines to the right the 1.25, $1.5,1.75$, etc thresholds for gains. The ratio profiles show the mean green to red ratio (red line) and the $95 \%$ confidence limits (yellow lines). Chromosome numbers are indicated. (C) Right, poorly differentiated DCIS and ductal invasive carcinoma grade 3; haematoxylin and eosin stained; magnification, $\times 5$. Right, examples of CGH ratio profiles of five chromosomes with significant differences in the preinvasive and invasive tumour component. Upper right, DCIS; lower right invasive carcinoma. 
COMPARATIVE GENOMIC HYBRIDISATION

$\mathrm{CGH}$ analysis and the criteria for the evaluation of copy number changes have been described elsewhere. ${ }^{2}$ In brief, $300 \mathrm{ng}$ of tumour DNA was labelled by a standard nick translation reaction with biotin-16-dUTP (Boehringer Mannheim, Mannheim, Germany). In addition, $300 \mathrm{ng}$ of reference DNA from a healthy female donor was labelled with digoxigenin-11-dUTP (Boehringer Mannheim). Labelled DNA fragments were purified from remaining nucleotides by column chromatography (Quiagen, Hilden, Germany). Repetitive sequences were blocked with $40 \mu \mathrm{g}$ of Cot 1 DNA.

The Cytovision 3.1 (Applied Imaging, Tyne and Wear, UK) software package was applied for digital image analysis and subsequent karyotyping.

E-CADHERIN IMMUNOHISTOCHEMISTRY

Immunohistochemistry was performed according to standard protocols, as described elsewhere. ${ }^{6}$

\section{Results}

LOBULAR CARCINOMA IN SITU AND ASSOCIATED LOBULAR CARCINOMA

On average, 2.6 copy number changes were detected in LCIS without evidence of amplifications. Four cases showed a loss of chromosomal material at $16 \mathrm{q}$, whereas losses of $11 \mathrm{q}$ were observed in two cases and, only sporadically, losses of $8 \mathrm{p}, 12 \mathrm{p}, 12 \mathrm{q}$, and the $\mathrm{X}$ chromosome. Gains of $1 \mathrm{q}$ were seen in four cases. The transition from LCIS to lobular invasive carcinoma was accompanied by different aberrations in each case, including gains of chromosomal material of $2 \mathrm{p}, 3 \mathrm{q}$, and $17 \mathrm{q}$, and losses of $1 \mathrm{p}, 17 \mathrm{p}$, and Xp. E-cadherin expression was weak to absent in all LCIS samples and their associated invasive counterparts.

INTERMEDIATELY DIFFERENTIATED DCIS AND ASSOCIATED CARCINOMA WITH PARTIAL OR COMPLETE LOBULAR DIFFERENTIATION

Three cases of DCIS showed identical profiles to their associated lobular invasive counterparts, one of them being a lobular invasive carcinoma of the pleomorphic subtype. In one case, two seperate localised areas of DCIS and the two associated classic lobular invasive counterparts could be analysed, showing on average 15 alterations, some of which could be demonstrated in all components (losses of $8 p$, $14 \mathrm{q}$, and $16 \mathrm{q}$ and gains of $8 \mathrm{q}$ and $12 \mathrm{p}$ ). The only alterations occurring in the invasive lesion were gains of $7 \mathrm{q}$ and $10 \mathrm{p}$. Figure 1 shows details of these alterations.

In three cases, a loss of E-cadherin expression or a weak, diffuse intracytoplasmic staining pattern in the invasive parts could be shown together with an intense membrane bound immunoreactivity in DCIS. In one case, DCIS and lobular invasive carcinoma showed a diffuse, weak, intracytoplasmic staining pattern.
POORLY DIFFERENTIATED DCIS IN CONTRAST TO ITS INVASIVE COUNTERPARTS

Four cases revealed identical profiles in the intraductal and invasive tumour parts. In one case, a normal profile for chromosome 13 could be detected in a DCIS area with a solid growth pattern, whereas $13 \mathrm{q}$ was lost in DCIS areas with a cribriform appearance and the invasive counterpart. In one case of a poorly differentiated DCIS, a 16q loss was observed that could not be detected in the invasive counterpart. One case showed a high degree of genetic heterogeneity involving chromosomes $3,6,7,12$, and 13 . An overview of this case is given in fig $1 \mathrm{C}$.

\section{Discussion}

It is generally accepted that DCIS and LCIS are precursor lesions of invasive breast cancer, both probably originating in the terminal ductal lobular unit. Nonetheless, distinguishing between both entities is sometimes difficult. This raised the question of whether these two tumour entities might have a common precursor. ${ }^{9}$ For many years, DCIS was seen as a uniform tumour group. However, clinical and molecular cytogenetic investigations revealed that DCIS might consist of different subgroups, which would justify a more detailed classification. ${ }^{27}{ }^{10}$ We were able to demonstrate two different pathways, with the loss of chromosomal material of $16 \mathrm{q}$ as a central event in well differentiated and intermediately differentiated DCIS. ${ }^{2}$ These results show a high degree of genetic homology between LCIS and well differentiated DCIS and, at least in part, confirm the results of Lu et al. ${ }^{4}$ Nevertheless there are controversial results such as the gain of $6 q$ and the loss of $16 p$ material. How far the use of a polymerase chain reaction based DNA enrichment technique (DOP-PCR) contributes to this remains unclear. We interpret our results as important evidence in favour of the hypothesis that LCIS and well differentiated DCIS reflect two closely related neoplastic lesions evolving from one neoplastic cell. The loss of E-cadherin expression probably represents a molecular switch to the loosely cohesive growth pattern of LCIS, with a similar cell morphology to that seen in well differentiated DCIS.

Against the background of a postulated progression from well differentiated DCIS to intermediately differentiated DCIS,${ }^{2}$ one could expect that there might be rare cases supporting the hypothesis that intermediately differentiated DCIS and invasive lobular carcinoma have a stem cell in common. In four cases of lobular invasive carcinoma, one of them of the pleomorphic subtype, we were able to show that there is a common stem cell origin for the invasive and preinvasive tumour components. This was further substantiated by the fact that lobular invasive carcinoma and intermediately differentiated DCIS show a high degree of genetic homology. ${ }^{11}{ }^{12}$ Immunohistochemical staining for E-cadherin in the DCIS component was noteworthy in that it showed a classic membrane bound staining pattern, whereas the invasive part showed a weak, diffuse, intracyto- 
Clonal proliferation

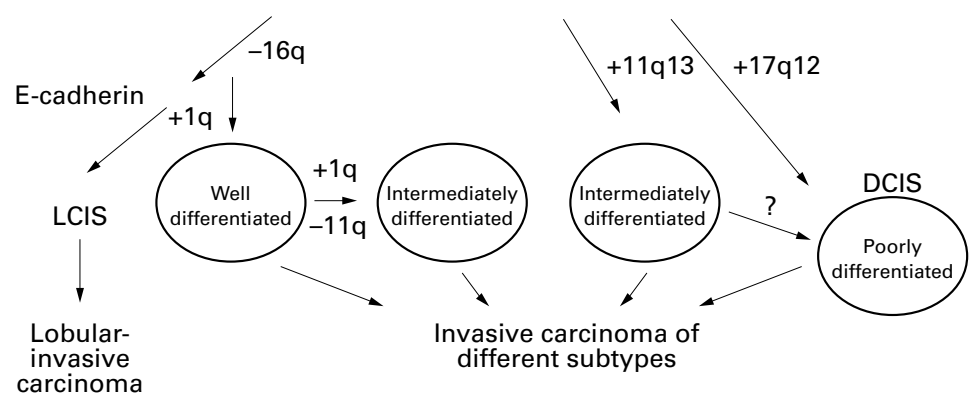

Figure 2 Hypothetical model of the pathogenesis of lobular carcinoma in situ (LCIS) and ductal carcinoma in situ (DCIS).

plasmic reaction. In addition, immunohistochemical investigations regarding $\mathrm{p} 53$ and HER-2/neu, as well as ploidy studies using flow cytometry, suggest that a subgroup of non-high grade DCIS cases share other genetic homologies with lobular invasive carcinoma. ${ }^{713}$

Comparisons of cumulative data from DCIS, LCIS, and invasive breast cancer have yielded that gains of $6 \mathrm{q}$ and $\mathrm{Xq}$ are associated with intralobular and intraductal growth, respectively. ${ }^{36}$ Allelic imbalances of $11 \mathrm{q} 13$ associated with a more aggressive phenotype have been described in DCIS and LCIS. ${ }^{214} 15$ Nevertheless, these alterations are not indicative of invasion because they were shown to be present in precursor lesions. Comparing preinvasive and invasive tumour components with CGH within one patient, ${ }^{23}$ heterogeneous findings were seen. Our investigations demonstrate that, by using $\mathrm{CGH}$ as a screening technique for chromosomal copy number changes, the step from intraductal to invasive breast cancer should not be seen as part of a linear progression. With DCIS showing, in some cases, fewer alterations than in invasive breast cancer, we have to postulate DCIS as a clonal proliferation with different cytogenetic subclones. This is in agreement with microsatellite analysis studies demonstrating a clear genetic diversity in preinvasive stages of breast cancer development and, furthermore, less intratumor diversity in carcinomas than in their preinvasive components. ${ }^{16}$ The same mechanism can be demonstrated in LCIS, with a loss of the X chromosome in one case of LCIS and a normal $\mathrm{CGH}$ ratio profile in the adjacent lobular invasive carcinoma, as shown in fig $1 \mathrm{~A}$. It seems unlikely that in LCIS the X chromosome is lost and is gained again during invasion. Therefore, it might be speculated that one of these subclones became invasive while one or more other subclones progressed intraductally. Therefore, progression to invasiveness cannot be attributed to a specific alteration, which is why further research on a sub-CGH level is needed. Interestingly, in one case, a $16 \mathrm{q}$ loss has been detected in a poorly differentiated DCIS, whereas the associated ductal invasive grade 3 carcinoma did not show any alterations in the CGH profile for this chromosome. This could be interpreted to mean that $16 \mathrm{q}$ losses in poorly differentiated breast lesions might be a secondary genetic event, as postulated earlier. ${ }^{12}$

Evidence exists that the overwhelming number of morphological forms of invasive breast cancer is reflected by specific genetic features. As a result of this, we propose a hypothetical progression model (fig 2). The underlying mechanism for the coexistence of ductal and lobular preinvasive and invasive tumours seems to be a proliferation of a single cell clone with two different forms of morphological appearance. Further studies will need to show whether transition from LCIS towards ductal invasive carcinoma or from DCIS towards lobular invasive carcinoma is possible. How far this hypothesis can be transferred to poorly differentiated tumours with mixed differentiation also remains a subject for intense investigation.

We are grateful to Ms K Poorthuis, Ms L Grote, and Ms U Neubert for excellent technical assistance and S Kölsch for editing the manuscript. Grants were provided by Deutsche Krebshilfe (10-1140-Bö-I) and the University of Münster (IMF; Bü 1198 31).

1 Teixeira MR, Pandis N, Bardi G, et al. Clonal heterogeneity in breast cancer: karyotypic comparisons of multiple intraand extra-tumorous samples from 3 patients. Int 7 Cancer 1995;63:63-8.

2 Buerger H, Otterbach F, Simon R, et al. Comparative genomic hybridization of ductal carcinoma in situ of the breast-evidence of multiple genetic pathways. $f$ Pathol 1999;187:396-402.

3 Kuukasjarvi T, Tanner M, Pennanen S, et al. Genetic changes in intraductal breast cancer detected by comparachanges in intraductal breast cancer detected by compara-
tive genomic hybridization. Am f Pathol 1997;150:146571.

$4 \mathrm{Lu}$ YJ, Osin P, Lakhani SR, et al. Comparative genomic hybridization analysis of lobular carcinoma in situ and atypical lobular hyperplasia and potential roles for gains and losses of genetic material in breast neoplasia. Cancer Res 1998;58:4721-7

5 Millikan R, Dressler L, Geradts J, et al. The need for epidemiologic studies of in-situ carcinoma of the breast. Breast Cancer Res Treat 1995;35:65-77.

6 Bankfalvi A, Terpe H-J, Breukelmann D, et al. Immunophenotypic and prognostic analysis of E-cadherin (E-Cad) and $\beta$-Catenin ( $\beta$-Cat) expression during breast carcinogenesis and tumour progression: a comparative study with CD44. Histopathology 1999;34:25-34.

7 Holland R, Peterse JL, Millis RR, et al. Ductal carcinoma in situ: a proposal for a new classification. Semin Diagn Pathol 994;11:167-80.

8 Ellis IO, Elston CW. Tumours of the breast. In: Fletcher CDM, ed. Diagnostic histopathology of tumours. Edinburgh: Churchill Livingstone, 1998:635-89.

9 Tavassoli FA. Pathology of the breast. Norwalk, Conneticut: Appleton and Lange, 1992

10 Silverstein MJ, Poller DN, Waisman JR, et al. Prognostic classification of breast ductal carcinoma-in-situ. Lancet 1995;345:1154-7.

11 Nishizaki T, Chew K, Chu L, et al. Genetic alterations in obular breast cancer by comparative genomic hybridization. Int $\mathcal{F}$ Cancer 1997;74:513-17.

12 Buerger H, Otterbach F, Simon R, et al. Different genetic pathways in the evolution of invasive breast cancer are associated with distinct morphological subtypes. F Pathol 1999;189:521-6.

13 Shackney SE, Shankey TV. Common patterns of genetic evolution in human solid tumors. Cytometry 1997;29:1-27.

14 Chuaqui RF, Zhuang Z, Emmert BM, et al. Analysis of loss Chuaqui RF, Zhuang Z, Emmert BM, et al. Analysis of loss
of heterozygosity on chromosome $11 \mathrm{q} 13$ in atypical ductal hyperplasia and in situ carcinoma of the breast. Am F Pathol 1997;150:297-303.

15 Nayar R, Zhuang Z, Merino MJ, et al. Loss of heterozygosity on chromosome $11 \mathrm{q} 13$ in lobular lesions of the breast using tissue microdissection and polymerase chain reaction. Hum Pathol 1997;28:277-82.

16 Fujii H, Marsh C, Cairns P, et al. Genetic divergence in the clonal evolution of breast cancer. Cancer Res 1996;56: 1493-7. 